\title{
OSCILLATION OF SYMPLECTIC DYNAMIC SYSTEMS
}

\author{
MARTIN BOHNER ${ }^{1}$ and ONDŘEJ DOŠLY' ${ }^{2}$
}

(Received 20 August, 2002; revised 1 December, 2002)

\begin{abstract}
We investigate oscillatory properties of a perturbed symplectic dynamic system on a time scale that is unbounded above. The unperturbed system is supposed to be nonoscillatory, and we give conditions on the perturbation matrix, which guarantee that the perturbed system becomes oscillatory. Examples illustrating the general results are given as well.
\end{abstract}

\section{Introduction}

We consider the symplectic dynamic system

$$
z^{\Delta}=\mathcal{S}(t) z
$$

that is, $\mathcal{S}$ is a symplectic and rd-continuous $2 n \times 2 n$ matrix-valued function and $z^{\Delta}$ is the delta derivative of $z$ (see Section 2 below and $[5,6,14]$ ), along with its perturbation

$$
z^{\Delta}=(\mathcal{S}(t)+\tilde{\mathcal{S}}(t)) z
$$

which is also supposed to be symplectic. Recall from [12] that $\mathcal{S}$ is called symplectic if

$$
\mathcal{S}^{T}(t) \mathcal{J}+\mathcal{J S}(t)+\mu(t) \mathcal{S}^{T}(t) \mathcal{J S}(t)=0 \quad \text { for all } t \in \mathbb{T},
$$

where $T$ is the time scale under consideration, $\mathcal{J}=\left(\begin{array}{cc}0 & 1 \\ -1 & 0\end{array}\right)$ and the superscript $T$ stands for the transpose of the matrix indicated. Since we are concerned with the oscillatory behaviour of the systems (1.1) and (1.2), we assume that $\mathbb{T}$ is unbounded

\footnotetext{
${ }^{1}$ University of Missouri-Rolla, Department of Mathematics and Statistics, 115 Rolla Building, Rolla, Missouri 65409-0020, USA; e-mail: bohner@umr.edu.

${ }^{2}$ Masaryk University Brno, Department of Mathematics, Faculty of Science, Janáčkovo nám. 2a, CZ-66295 Bmo, Czech Republic; e-mail: dosly@math.muni.cz.

(C) Australian Mathematical Society 2004, Serial-fee code 1446-1811/04
} 
above. After a short introduction in Section 2 on time scales calculus for those readers unfamiliar with that topic, we collect in Section 3 the main properties and definitions connected with symplectic systems. Section 4 is then devoted to the main results of this paper. Assuming that (1.1) is nonoscillatory, we give conditions that imply that the perturbed system (1.2) is oscillatory. The concluding Section 5 contains some examples illustrating the main results of the paper.

Our results are quite general as systems (1.1) contain a variety of important systems as special cases, for example, linear Hamiltonian differential systems, linear Hamiltonian difference systems, Sturm-Liouville differential equations (of any order), Sturm-Liouville difference equations (of any order), self-adjoint matrix differential systems, self-adjoint matrix difference systems and symplectic difference systems. Our oscillation criteria presented in Section 4 are new even in many of these special cases, as will be seen in the last section.

\section{Preliminaries about time scales}

In this short section we give some preliminaries on time scales for those readers unfamiliar with the calculus recently developed by Stefan Hilger [14] (see [5, 6]). A time scale $T$ is an arbitrary nonempty closed subset of the reals (unbounded above for the purpose of this paper), and the associated forward jump operator $\sigma: \mathbb{T} \rightarrow \mathbb{T}$ and the graininess $\mu: \mathbb{T} \rightarrow[0, \infty)$ are defined by

$$
\sigma(t):=\inf \{s \in \mathbb{T}: s>t\} \text { and } \mu(t)=\sigma(t)-t .
$$

A point $t \in \mathbb{T}$ with $\sigma(t)=t$ is called right-dense while $t$ is referred to as being rightscattered if $\sigma(t)>t$. The backward jump operator and left-dense and left-scattered points are defined in a similar way. A function $f: \mathbb{T} \rightarrow \mathbb{R}$ is said to be $r d$-continuous, denoted by $f \in \mathrm{C}_{\mathrm{rd}}$, if it is continuous at each right-dense point and if there exists a finite left limit in all left-dense points. The (delta) derivative $f^{\Delta}$ of $f$ is defined by

$$
f^{\Delta}(t)=\lim _{\substack{s \rightarrow t^{t} \\ s \in U(t)}} \frac{f(\sigma(t))-f(s)}{\sigma(t)-s}, \quad \text { where } \quad U(t)=\mathbb{T} \backslash\{\sigma(t)\}
$$

The derivative and the shift operator are related by the useful formula

$$
f^{\sigma}=f+\mu f^{\Delta}, \text { where } f^{\sigma}:=f \circ \sigma .
$$

We will also make use of the following product and quotient rules for the derivative of the product $f g$ and the quotient $f / g$ (where $g g^{\sigma} \neq 0$ ) of two differentiable functions $f$ and $g$ :

$$
(f g)^{\Delta}=f^{\Delta} g+f^{\sigma} g^{\Delta} \text { and }\left(\frac{f}{g}\right)^{\Delta}=\frac{f^{\Delta} g-f g^{\Delta}}{g g^{\sigma}}
$$


For $a, b \in \mathbb{U}$ and a differentiable function $f$, the Cauchy integral of $f^{\Delta}$ is defined by

$$
\int_{a}^{b} f^{\Delta}(t) \Delta t=f(b)-f(a),
$$

and improper integrals are defined as

$$
\int_{a}^{\infty} f(s) \Delta s=\lim _{t \rightarrow \infty} \int_{a}^{t} f(s) \Delta s .
$$

Note that rd-continuous functions possess antiderivatives and hence are integrable. Hilger's existence theorem for initial value problems with first-order linear equations says that, given an rd-continuous and regressive (that is, $I+\mu(t) P(t)$ is invertible for all $t \in \mathbb{T}$ ) matrix-valued function $P$, there is exactly one solution of the dynamic system $z^{\Delta}=P(t) z$ that satisfies a given initial condition $z\left(t_{0}\right)=z_{0}$.

EXAMPLE 2.1. In case $\mathbb{T}=\mathbb{R}$ we have

$$
\sigma(t)=t, \quad \mu(t) \equiv 0, \quad f^{\Delta}=f^{\prime}, \quad \int_{a}^{b} f(t) \Delta t=\int_{a}^{b} f(t) d t,
$$

and symplectic (differential) systems (1.1) are of the form (note $\mu(t) \equiv 0$ in (1.3))

$$
z^{\prime}=\mathcal{H}(t) z, \quad \text { where } \mathcal{J H} \text { is symmetric, that is, } \mathcal{J H}=\mathcal{H}^{T} \mathcal{J}^{T}
$$

(these are the so-called linear Hamiltonian differential systems). In the case when $\mathbb{T}=\mathbb{Z}$ we have (where we denote the forward difference by $\Delta f(t)=f(t+1)-f(t)$ )

$$
\sigma(t)=t+1, \quad \mu(t) \equiv 1, \quad f^{\Delta}=\Delta f, \quad \int_{a}^{b} f(t) \Delta t=\sum_{t=a}^{b-1} f(t),
$$

and symplectic difference systems (1.1) are of the form (note $\mu(t) \equiv 1$ in (1.3))

$$
z(t+1)=\mathcal{S}(t) z(t), \quad \text { where } \mathcal{S} \text { is symplectic, that is, } \mathcal{S}^{T} \mathcal{J S}=\mathcal{J} .
$$

Note that while these two examples nicely exhibit the unification feature of time scales calculus, the extension feature of this calculus can be seen from using an arbitrary nonempty closed subset of the reals as the time scale, for example, the set of all nonnegative integer powers of a number $q>1$ (note that $\sigma(t)=q t$ in this case, and dynamic equations on such time scales are called $q$-difference equations), a union of closed intervals, the Cantor set, the set of all squares (or roots or cubes etc.) of nonnegative integers, the set of all integer multiples of a number $h>0$, the set of all harmonic numbers, and many more. 


\section{Basic properties of symplectic systems}

In this section we recall some basic facts concerning symplectic dynamic systems (1.1). As mentioned in the previous section, a symplectic dynamic system is a firstorder linear dynamic system whose coefficient matrix satisfies (1.3). This identity implies that the matrix $I+\mu(t) \mathcal{S}(t)$ is symplectic for each $t \in \mathbb{T}$, that is,

$$
(I+\mu \mathcal{S})^{T} \mathcal{J}(I+\mu \mathcal{S})=\mathcal{J}
$$

holds on $\pi$. This last identity is equivalent to $(I+\mu \mathcal{S}) \mathcal{J}(I+\mu \mathcal{S})^{T}=\mathcal{J}$, so a symplectic dynamic system can be also characterised as a system (1.1) whose coefficient matrix satisfies

$$
\mathcal{S}(t) \mathcal{J}+\mathcal{J S}^{T}(t)+\mu(t) \mathcal{S}(t) \mathcal{J} \mathcal{S}^{T}(t)=0 \quad \text { for all } t \in \mathbb{T} .
$$

If we write $\mathcal{S}=\left(\begin{array}{cc}A & B \\ C & D\end{array}\right)$ with $n \times n$ matrix-valued functions $A, B, C$ and $D$, then (1.3) and (3.1) read

$$
\left\{\begin{aligned}
C-C^{T}+\mu\left(A^{T} C-C^{T} A\right) & =0, & C-C^{T}+\mu\left(C D^{T}-D C^{T}\right) & =0 \\
B^{T}-B+\mu\left(B^{T} D-D^{T} B\right) & =0, & B^{T}-B+\mu\left(A B^{T}-B A^{T}\right) & =0 \\
A^{T}+D+\mu\left(A^{T} D-C^{T} B\right) & =0, & A+D^{T}+\mu\left(A D^{T}-B C^{T}\right) & =0 .
\end{aligned}\right.
$$

Next, if $Z$ and $\tilde{Z}$ are two $2 n \times n$ matrix-valued solutions of (1.1), then $Z^{T} \mathcal{J} \tilde{Z}$ is a constant $n \times n$ matrix (this is a so-called Wronskian-type identity). A solution $Z$ is said to be a conjoined basis if rank $Z \equiv n$ and $Z^{T} \mathcal{J} Z \equiv 0$. Oscillatory properties of (1.1) are defined using the concept of focal points. A $2 n \times n$ matrix-valued solution $Z$ of (1.1) has no focal point in the interval $\mathcal{I}=(a, b] \subset \mathbb{T}$ if $X(t)$ is invertible at all dense points $t \in \mathcal{I}$ and if

$$
\operatorname{Ker} X^{\sigma}(t) \subset \operatorname{Ker} X(t) \quad \text { and } \quad X(t)\left(X^{\sigma}(t)\right)^{\dagger} B(t) \geq 0
$$

on $\mathcal{I}^{\kappa}$ (here, $\dagger$ denotes the Moore-Penrose generalised inverse). The system (1.1) is called disconjugate on $\mathcal{I}$ if the solution $Z=\left(\begin{array}{l}X \\ U\end{array}\right)$ given by the initial condition $X(a)=0$ and $U(a)=I$ (the so-called principal solution of (1.1) at $a$ ) has no focal points in $\mathcal{I}$. System (1.1) is called nonoscillatory if there exists $T \in \mathbb{T}$ such that it is disconjugate on $\left(T, T_{1}\right]$ for every $T_{1}>T$, and it is said to be oscillatory in the opposite case.

In our treatment we will also need the concept of the principal and nonprincipal solution of (1.1) at $\infty$ as introduced in [11] and studied in [3]. System (1.1) is said to be eventually controllable if the trivial solution $z=\left(\begin{array}{l}x \\ u\end{array}\right) \equiv\left(\begin{array}{l}0 \\ 0\end{array}\right)$ is the only solution for which $x \equiv 0$ eventually. If (1.1) is eventually controllable and nonoscillatory, then 
the first component $X$ of any conjoined basis $Z=\left(\begin{array}{l}X \\ U\end{array}\right)$ is eventually nonsingular, and for every $T \in \mathbb{T}$ there exists $t_{1}>T$ such that the matrix

$$
\int_{T}^{t}\left(X^{\sigma}\right)^{-1}(\tau) B(\tau)\left(X^{T}\right)^{-1}(\tau) \Delta \tau
$$

is positive definite whenever $t>t_{1}$. Among all conjoined bases of an eventually controllable and nonoscillatory symplectic dynamic system one can distinguish the so-called principal solution at $\infty$, which is the conjoined basis $\tilde{Z}=\left(\begin{array}{l}\bar{X} \\ \tilde{U}\end{array}\right)$ with the property that

$$
\lim _{t \rightarrow \infty} X^{-1}(t) \tilde{X}(t)=0
$$

for any conjoined basis $Z=\left(\begin{array}{l}x \\ U\end{array}\right)$ for which the (constant) matrix $Z^{T} \mathcal{J} \tilde{Z}$ is nonsingular. Any conjoined basis $Z=\left(\begin{array}{l}X \\ U\end{array}\right)$ for which $Z^{T} \mathcal{J} \tilde{Z}$ is a nonsingular matrix is called a nonprincipal solution at $\infty$. Note that the principal solution at $\infty$ is uniquely determined up to a right multiplicative constant nonsingular $n \times n$ matrix factor, and that (3.3) is equivalent to

$$
\lim _{t \rightarrow \infty}\left(\int^{t}\left(X^{\sigma}\right)^{-1}(\tau) B(\tau)\left(X^{T}\right)^{-1}(\tau) \Delta \tau\right)^{-1}=0 .
$$

When investigating oscillatory properties of (1.1), a fundamental rôle is played by the so-called Reid roundabout theorem, which relates oscillatory properties of (1.1) to solvability of a certain associated Riccati-type equation and to positivity of the quadratic functional

$$
\mathcal{F}(z ; a, b):=\int_{a}^{b} z^{T}(\tau)\left\{\mathcal{S}^{T}(\tau) \mathcal{K}+\mathcal{K} \mathcal{S}(\tau)+\mu(\tau) \mathcal{S}^{T}(\tau) \mathcal{K} \mathcal{S}(\tau)\right\} z(\tau) \Delta \tau
$$

with $\mathcal{K}=\left(\begin{array}{ll}0 & 0 \\ 1 & 0\end{array}\right)$, over the class of pairs $z=\left(\begin{array}{l}x \\ u\end{array}\right)$ such that $\mathcal{K} z^{\Delta}=\mathcal{K} \mathcal{S}(t) z$ and $x(a)=x(b)=0$. This roundabout theorem for (1.1) was established in the recent paper [16]. Here we use only a part of this roundabout theorem, which is formulated in the next proposition (in a slightly modified form; compare with [12] or [16]).

PROPOSITION 3.1. Suppose that for every $T \in \mathbb{T}$ there exists a pair $z=\left(\begin{array}{l}x \\ u\end{array}\right)$ such that $x \in \mathrm{C}_{\mathrm{rd}}^{1}[T, \infty), u \in \mathrm{C}_{\mathrm{rd}}[T, \infty)$ piecewise, $x^{\Delta}=A(t) x+B(t) u$, supp $x \subset[T, \infty)$ (that is, $x(T)=0$ and there exists $T_{1}>T$ such that $x(t) \equiv 0$ for $t>T_{1}$ ) and

$$
\mathcal{F}(z ; T, \infty)=\int_{T}^{\infty} z^{T}(\tau)\left\{\mathcal{S}^{T} \mathcal{K}+\mathcal{K} \mathcal{S}+\mu \mathcal{S}^{T} \mathcal{K} \mathcal{S}\right\}(\tau) z(\tau) \Delta \tau<0
$$

Then (1.1) is oscillatory. 
Note also that for $\mathcal{S}=\left(\begin{array}{cc}A & B \\ C & D\end{array}\right)$ and $z=\left(\begin{array}{l}x \\ u\end{array}\right)$ the functional $\mathcal{F}(z ; a, b)$ takes the form

$$
\mathcal{F}(x, u ; a, b)=\int_{a}^{b}\left\{\left(\begin{array}{l}
x \\
u
\end{array}\right)^{T}\left(\begin{array}{cc}
C^{T}(I+\mu A) & \mu C^{T} B \\
\mu B^{T} C & (I+\mu D)^{T} B
\end{array}\right)\left(\begin{array}{l}
x \\
u
\end{array}\right)\right\}(\tau) \Delta \tau .
$$

We conclude this section with a result concerning a certain transformation of (1.1); see [12]. Let $H, K: \mathbb{T} \rightarrow \mathbb{R}^{n \times n}$ be $C_{\mathrm{rd}}^{1}$-matrices such that $H$ is nonsingular and $H^{T} K=K^{T} H$, that is, the matrix $\mathcal{R}=\left(\begin{array}{l}{ }_{K}^{H} \\ \left(H^{T}\right)^{-1}\end{array}\right)$ is symplectic. Consider the transformation $z=\mathcal{R} \bar{z}$ of the symplectic dynamic system (1.1), which transforms (1.1) into the system

$$
\bar{z}^{\Delta}=\overline{\mathcal{S}}(t) \bar{z} \quad \text { with } \quad \overline{\mathcal{S}}=\left(\begin{array}{ll}
\bar{A} & \bar{B} \\
\bar{C} & \bar{D}
\end{array}\right)
$$

which is again symplectic, and the matrices $\bar{A}, \bar{B}, \bar{C}$ and $\bar{D}$ are given by the formulas

$$
\begin{aligned}
& \bar{A}=\left(H^{\sigma}\right)^{-1}\left(A H+B K-H^{\Delta}\right) \\
& \bar{B}=\left(H^{\sigma}\right)^{-1} B\left(H^{T}\right)^{-1} \\
& \bar{C}=\left(K^{\sigma}\right)^{T}\left(H^{\Delta}-A H-B K\right)-\left(H^{\sigma}\right)^{T}\left(K^{\Delta}-C H-D K\right) \\
& \bar{D}=\left(H^{\Delta}-D^{T} H^{\sigma}-B^{T} K^{\sigma}\right)^{T}\left(H^{T}\right)^{-1}
\end{aligned}
$$

Consequently, if $\left(\begin{array}{l}x \\ U\end{array}\right)$ is a solution of (1.1) such that $X$ is nonsingular, setting $H=X$ and $K=U$, we have $\bar{A}=0$ and $\bar{C}=0$ (this is obvious) and $\bar{D}=0$ (this follows from the fact that (3.5) is again symplectic, that is, (3.2) hold for $\bar{A}, \bar{B}, \bar{C}$ and $\bar{D}$ ).

\section{Oscillation criteria}

Throughout we assume that the perturbation matrix $\tilde{\mathcal{S}}$ from (1.2) is of the form

$$
\tilde{\mathcal{S}}=\left(\begin{array}{cc}
0 & 0 \\
W(I+\mu A) & \mu W B
\end{array}\right) \text {. }
$$

Let us briefly explain why we choose $\tilde{\mathcal{S}}$ of the form (4.1). First we require that the admissibility equation for the quadratic functional corresponding to (1.2), $\mathcal{K} z^{\Delta}=$ $\mathcal{K}(\mathcal{S}+\tilde{\mathcal{S}}) z$, is independent of $\tilde{\mathcal{S}}$, that is, $\mathcal{K} \tilde{\mathcal{S}}_{z}=0$ and hence $\tilde{\mathcal{S}}=\left(\begin{array}{cc}0 & 0 \\ \hat{c} & \hat{D}\end{array}\right)$. This requirement is perhaps not strictly necessary, but it is reasonable from the application point of view as we will see in the last section. Another requirement is that the perturbed system (1.2) is again a symplectic dynamic system, that is, (1.3) and (3.1) 
must hold. This means that

$$
\left\{\begin{array}{l}
\hat{C}^{T}(I+\mu A)=(I+\mu A)^{T} \hat{C} \\
(I+\mu A) \hat{C}^{T}=\mu B \hat{D}^{T} \\
\mu \hat{D}^{T} B=\mu B^{T} \hat{D} \\
\hat{C}\left(I+\mu D^{T}\right)-(I+\mu D) \hat{C}^{T}=\mu\left(\hat{D} C^{T}-C \hat{D}^{T}\right) \\
\hat{D}^{T}(I+\mu A)=\mu B^{T} \hat{C} .
\end{array}\right.
$$

If $\mu=0$, then obviously $\hat{D}=0$ and $\hat{C}=W$ is a symmetric matrix. Now suppose $\mu \neq 0$. Then the fact that $I+\mu \mathcal{S}$ is symplectic implies

$$
(I+\mu A)(I+\mu D)^{T}-\mu^{2} B C^{T}=I \quad \text { and } \quad \operatorname{rank}(I+\mu A, \mu B)=n .
$$

Hence, since $(I+\mu A) \mu B^{T}=\mu B(I+\mu A)^{T}$,

$$
\operatorname{Ker}(I+\mu A, \mu B)=\operatorname{Im}\left(\begin{array}{c}
\mu B^{T} \\
-(I+\mu A)^{T}
\end{array}\right) .
$$

Now, the second identity in (4.2) implies that

$$
\left(\begin{array}{c}
\hat{C} \\
-\hat{D}
\end{array}\right) \in \operatorname{Im}\left(\begin{array}{c}
\mu B^{T} \\
-(I+\mu A)^{T}
\end{array}\right)
$$

that is, there exists an $n \times n$ matrix $W$ such that

$$
\hat{D}^{T}=\mu B^{T} W \quad \text { and } \quad \hat{C}^{T}=(I+\mu A)^{T} W .
$$

Substituting this into (4.2), we find that $W$ must be symmetric, and then all identities in (4.2) are satisfied.

Now we are ready to present our oscillation criteria for systems (1.2). We first give conditions that imply, assuming nonoscillation of (1.1), that the perturbed system (1.2) is oscillatory.

THEOREM 4.1. Suppose that (1.1) is nonoscillatory and eventually controllable, and let $\left(\begin{array}{l}X \\ U\end{array}\right)$ be its principal solution at $\infty$. If

$$
W(t) \leq 0 \text { for large } t \in \mathbb{T}
$$

and if there exists a pair $\left(\begin{array}{c}\bar{x} \\ \bar{u}\end{array}\right): \mathbb{T} \rightarrow \mathbb{R}^{2 n}$ such that

$$
\tilde{x} \in \mathrm{C}_{\mathrm{rd}}^{1}, \quad \tilde{u} \in \mathrm{C}_{\mathrm{rd}}, \quad \tilde{x}^{\Delta}=\left(X^{\sigma}\right)^{-1} B\left(X^{T}\right)^{-1} \tilde{u}
$$

and

$$
\int^{\infty}\left\{\tilde{u}^{T}\left(X^{\sigma}\right)^{-1} B\left(X^{T}\right)^{-1} \tilde{u}+\left(\tilde{x}^{\sigma}\right)^{T}\left(X^{\sigma}\right)^{T} W X^{\sigma} \tilde{x}^{\sigma}\right\}(\tau) \Delta \tau=-\infty,
$$

then (1.2) is oscillatory. 
PROOF. Consider the transformation $z=\mathcal{R} \tilde{z}$ of (1.2) with $\mathcal{R}=\left(\begin{array}{ll}X & 0 \\ U & \left(x^{T}\right)^{-1}\end{array}\right)$. This transformation preserves the oscillatory behaviour of (1.2) and transforms (1.2) into

$$
\tilde{z}^{\Delta}=\left(\begin{array}{cc}
\frac{0}{W} & \mu \bar{B} \\
\bar{W} & \bar{B}
\end{array}\right) \tilde{z}
$$

where $\bar{B}=\left(X^{\sigma}\right)^{-1} B\left(X^{T}\right)^{-1}$ and $\bar{W}=\left(X^{\sigma}\right)^{T} W X^{\sigma}$. To prove that (4.6) is oscillatory (and hence that (1.2) is oscillatory), according to Proposition 3.1 it suffices to construct for every $T \in \mathbb{T}$ a pair $z=\left(\begin{array}{l}x \\ u\end{array}\right)$ such that $x^{\Delta}=\bar{B} u, x \in C_{\mathrm{rd}}^{1}, u \in \mathrm{C}_{\mathrm{rd}}$ piecewise on $[T, \infty)$, supp $x \subset[T, \infty)$ and $\widetilde{\mathcal{F}}(x, u)<0$, where

$$
\begin{aligned}
\tilde{\mathcal{F}}(x, u) & =\int_{T}^{\infty}\left\{\left(\begin{array}{l}
x \\
u
\end{array}\right)^{T}\left(\begin{array}{cc}
\bar{W} & \mu \bar{W} \bar{B} \\
\mu \bar{B}^{T} \bar{W} & \bar{B}+\mu^{2} \bar{B}^{T} \bar{W} \bar{B}
\end{array}\right)\left(\begin{array}{l}
x \\
u
\end{array}\right)\right\}(\tau) \Delta \tau \\
& =\int_{T}^{\infty}\left\{u^{T} \bar{B} u+(x+\mu \bar{B} u)^{T} \bar{W}(x+\mu \bar{B} u)\right\}(\tau) \Delta \tau \\
& =\int_{T}^{\infty}\left\{u^{T} \bar{B} u+\left(x^{\sigma}\right)^{T} \bar{W} x^{\sigma}\right\}(\tau) \Delta \tau .
\end{aligned}
$$

Define the pair $\left(\begin{array}{l}x \\ u\end{array}\right)$ by

$$
\left(\begin{array}{l}
x \\
u
\end{array}\right)= \begin{cases}\left(\begin{array}{l}
0 \\
0
\end{array}\right) & \text { if } t \leq T \\
\left(\begin{array}{l}
x_{1} \\
u_{1}
\end{array}\right) & \text { if } t \in\left[T, t_{1}\right] \\
\left(\begin{array}{l}
x \\
\tilde{u}
\end{array}\right) & \text { if } t \in\left[t_{1}, t_{2}\right] \\
\left(\begin{array}{l}
x_{2} \\
u_{2}
\end{array}\right) & \text { if } t \in\left[t_{2}, t_{3}\right] \\
\left(\begin{array}{l}
0 \\
0
\end{array}\right) & \text { if } t \geq t_{3},\end{cases}
$$

where $T \in \mathbb{T}$ is arbitrary, $t_{3}>t_{2}>t_{1}>T$ will be specified later and $\left(\begin{array}{l}x_{1} \\ u_{1}\end{array}\right)$ and $\left(\begin{array}{l}x_{2} \\ u_{2}\end{array}\right)$ are solutions of $x^{\Delta}=\bar{B} u$ satisfying $x_{1}(T)=0, x_{1}\left(t_{1}\right)=\tilde{x}\left(t_{1}\right), x_{2}\left(t_{2}\right)=\tilde{x}\left(t_{2}\right)$ and $x_{2}\left(t_{3}\right)=0$, that is,

$$
\begin{aligned}
& x_{1}(t)=\left(\int_{T}^{t} \bar{B}(\tau) \Delta \tau\right)\left(\int_{T}^{t_{1}} \bar{B}(\tau) \Delta \tau\right)^{-1} \tilde{x}\left(t_{1}\right), \\
& u_{1}(t)=\left(\int_{T}^{t_{1}} \bar{B}(\tau) \Delta \tau\right)^{-1} \tilde{x}\left(t_{1}\right) \\
& x_{2}(t)=\left(\int_{t}^{t_{3}} \bar{B}(\tau) \Delta \tau\right)\left(\int_{t_{2}}^{t_{3}} \bar{B}(\tau) \Delta \tau\right)^{-1} \tilde{x}\left(t_{2}\right), \\
& u_{2}(t)=-\left(\int_{t_{2}}^{t_{3}} \bar{B}(\tau) \Delta \tau\right)^{-1} \tilde{x}\left(t_{2}\right) .
\end{aligned}
$$


Note that controllability of (1.1) implies that $\int_{T}^{t} \bar{B}(\tau) \Delta \tau$ is really invertible if $t$ is sufficiently large. Then

$$
\begin{aligned}
\tilde{\mathcal{F}}(x, u)= & \int_{T}^{t_{1}}\left\{u_{1}^{T} \bar{B} u_{1}+\left(x_{1}^{\sigma}\right)^{T} \bar{W} x_{1}^{\sigma}\right\}(\tau) \Delta \tau+\int_{t_{1}}^{t_{2}}\left\{\tilde{u}^{T} \bar{B} \tilde{u}+\left(\tilde{x}^{\sigma}\right)^{T} \bar{W} \tilde{x}^{\sigma}\right\}(\tau) \Delta \tau \\
& +\int_{t_{2}}^{t_{3}}\left\{u_{2}^{T} \bar{B} u_{2}+\left(x_{2}^{\sigma}\right)^{T} \bar{W} x_{2}^{\sigma}\right\}(\tau) \Delta \tau \\
\leq & \tilde{x}^{T}\left(t_{1}\right)\left(\int_{T}^{t_{1}} \bar{B}(\tau) \Delta \tau\right)^{-1} \tilde{x}\left(t_{1}\right)+\tilde{x}^{T}\left(t_{2}\right)\left(\int_{t_{2}}^{t_{3}} \bar{B}(\tau) \Delta \tau\right) \tilde{x}\left(t_{2}\right) \\
& +\int_{t_{1}}^{t_{2}}\left\{\tilde{u}^{T} \bar{B} \tilde{u}+\left(\tilde{x}^{\sigma}\right)^{T} \bar{W} \tilde{x}^{\sigma}\right\}(\tau) \Delta \tau .
\end{aligned}
$$

Here we have used (4.3). Now let $\varepsilon>0$ be arbitrary and $t_{1}>T$ be fixed. According to (4.5), $t_{2}>t_{1}$ can be chosen in such a way that

$$
\int_{t_{1}}^{t_{2}}\left\{\tilde{u}^{T} \bar{B} \tilde{u}+\left(\tilde{x}^{\sigma}\right)^{T} \bar{W} \tilde{x}^{\sigma}\right\}(\tau) \Delta \tau<-\tilde{x}_{1}^{T}\left(t_{1}\right)\left(\int_{T}^{t_{1}} \bar{B}(\tau) \Delta \tau\right)^{-1} \tilde{x}_{1}\left(t_{1}\right)-\varepsilon .
$$

Finally, since $\left(\begin{array}{l}X \\ U\end{array}\right)$ is the principal solution of (1.1), we have

$$
\left(\int_{t_{2}}^{t} \bar{B}(\tau) \Delta \tau\right)^{-1}=\left(\int_{t_{2}}^{t}\left\{\left(X^{\sigma}\right)^{-1} B\left(X^{T}\right)^{-1}\right\}(\tau) \Delta \tau\right)^{-1} \rightarrow 0 \quad \text { as } t \rightarrow \infty,
$$

and hence $t_{3}$ can be chosen such that $\tilde{x}_{2}^{T}\left(t_{2}\right)\left(\int_{t_{2}}^{t_{3}} \bar{B}(\tau) \Delta \tau\right)^{-1} \tilde{x}_{2}\left(t_{2}\right)<\varepsilon$. Summarising the previous computations we see that $\widetilde{\mathcal{F}}(x, u)<0$ if $T<t_{1}<t_{2}<t_{3}$ are chosen as above, and hence (4.6) is oscillatory. This means that (1.2) is oscillatory as well.

Our next result offers another oscillation criterion for (1.2).

THEOREM 4.2. Suppose that (4.3) holds and let $\left(\begin{array}{l}x \\ U\end{array}\right)$ and $\left(\begin{array}{l}\tilde{x} \\ \tilde{u}\end{array}\right)$ be as in Theorem 4.1; however, instead of (4.5) we assume that the integral

$$
\int^{\infty}\left\{\tilde{u}^{T} \bar{B} \tilde{u}+\left(\tilde{x}^{\sigma}\right)^{T} \bar{W} \tilde{x}^{\sigma}\right\}(\tau) \Delta \tau
$$

is convergent. Moreover, we suppose that

If

$$
\tilde{x}^{T}(t)\left(\int^{t} \bar{B}(\tau) \Delta \tau\right)^{-1} \tilde{x}(t) \rightarrow 0 \text { as } t \rightarrow \infty .
$$

$$
\limsup _{t \rightarrow \infty} \frac{\int_{t}^{\infty}\left\{\tilde{u}^{T} \bar{B} \tilde{u}+\left(\tilde{x}^{\sigma}\right)^{T} \bar{W} \tilde{x}^{\sigma}\right\}(\tau) \Delta \tau}{\tilde{x}^{T}(t)\left(\int^{t} \bar{B}(\tau) \Delta \tau\right)^{-1} \tilde{x}(t)}<-1,
$$

then (1.2) is oscillatory. 
PROOF. First note that the lower limit of integration in the integral in the denominator of (4.8) is not important. Indeed, since $\left(\int^{t} \bar{B}(\tau) \Delta \tau\right)^{-1} \rightarrow 0$ as $t \rightarrow \infty$ and (4.7) holds, we find for any $a, b \in \mathbb{T}$

$$
\lim _{t \rightarrow \infty} \frac{\tilde{x}^{T}(t)\left(\int_{a}^{t} \bar{B}(\tau) \Delta \tau\right)^{-1} \tilde{x}(t)}{\tilde{x}^{T}(t)\left(\int_{b}^{t} \bar{B}(\tau) \Delta \tau\right)^{-1} \tilde{x}(t)}=1 .
$$

We use the same $\left(\begin{array}{l}x \\ u\end{array}\right)$ as in the proof of Theorem 4.1. Using the computation given there, we have

$$
\begin{aligned}
\tilde{\mathcal{F}}(x, u) \leq & \tilde{x}^{T}\left(t_{1}\right)\left(\int_{T}^{t_{1}} \bar{B}(\tau) \Delta \tau\right)^{-1} \tilde{x}\left(t_{1}\right)+\tilde{x}^{T}\left(t_{2}\right)\left(\int_{t_{2}}^{t_{3}} \bar{B}(\tau) \Delta \tau\right)^{-1} \tilde{x}\left(t_{2}\right) \\
& +\int_{t_{1}}^{t_{2}}\left\{\tilde{u}^{T} \bar{B} \tilde{u}+\left(\tilde{x}^{\sigma}\right)^{T} \bar{W} \tilde{x}^{\sigma}\right\}(\tau) \Delta \tau \\
= & \tilde{x}^{T}\left(t_{1}\right)\left(\int_{T}^{t_{1}} \bar{B}(\tau) \Delta \tau\right)^{-1} \tilde{x}\left(t_{1}\right)\left\{1+\Gamma_{1}+\Gamma_{2}\right\}
\end{aligned}
$$

where

$$
\Gamma_{1}:=\frac{\int_{t_{1}}^{t_{2}}\left\{\tilde{u}^{T} \bar{B} \tilde{u}+\left(\tilde{x}^{\sigma}\right)^{T} \bar{W} \tilde{x}^{\sigma}\right\}(\tau) \Delta \tau}{\tilde{x}^{T}\left(t_{1}\right)\left(\int_{T}^{t_{1}} \bar{B}(\tau) \Delta \tau\right)^{-1} \tilde{x}\left(t_{1}\right)} \quad \text { and } \quad \Gamma_{2}:=\frac{\tilde{x}^{T}\left(t_{2}\right)\left(\int_{t_{2}}^{t_{3}} \bar{B}(\tau) \Delta \tau\right)^{-1} \tilde{x}\left(t_{2}\right)}{\tilde{x}^{T}\left(t_{1}\right)\left(\int_{T}^{t_{1}} \bar{B}(\tau) \Delta \tau\right)^{-1} \tilde{x}\left(t_{1}\right)} \text {. }
$$

Now, let $\varepsilon>0$ be such that the limit superior in (4.8) is less than $-1-3 \varepsilon$. The point $t_{1}>T$ is now chosen such that

$$
\frac{\int_{t_{1}}^{\infty}\left\{\tilde{u}^{T} \bar{B} \tilde{u}+\left(\tilde{x}^{\sigma}\right)^{T} \bar{W} \tilde{x}^{\sigma}\right\}(\tau) \Delta \tau}{\tilde{x}^{T}\left(t_{1}\right)\left(\int_{T}^{t_{1}} \bar{B}(\tau) \Delta \tau\right)^{-1} \tilde{x}\left(t_{1}\right)}<-1-2 \varepsilon
$$

and $t_{2}>t_{1}$ such that

$$
\Gamma_{1}=\frac{\int_{t_{1}}^{t_{2}}\left\{\tilde{u}^{T} \bar{B} \tilde{u}+\left(\tilde{x}^{\sigma}\right)^{T} \bar{W} \tilde{x}^{\sigma}\right\}(\tau) \Delta \tau}{\tilde{x}^{T}\left(t_{1}\right)\left(\int_{\tau}^{t_{1}} \bar{B}(\tau) \Delta \tau\right)^{-1} \tilde{x}\left(t_{1}\right)}<-1-\varepsilon
$$

Finally, we take $t_{3}>t_{2}$ such that

$$
\Gamma_{2}=\frac{\tilde{x}^{T}\left(t_{2}\right)\left(\int_{t_{2}}^{t_{3}} \bar{B}(\tau) \Delta \tau\right)^{-1} \tilde{x}\left(t_{2}\right)}{\tilde{x}^{T}\left(t_{1}\right)\left(\int_{T}^{t_{1}} \bar{B}(\tau) \Delta \tau\right)^{-1} \tilde{x}\left(t_{1}\right)}<\varepsilon
$$

This is possible since $\left(\int_{t_{2}}^{t} \bar{B}(\tau) \Delta \tau\right)^{-1} \rightarrow 0$ as $t \rightarrow \infty$. Altogether, for these $t_{3}>$ $t_{2}>t_{1}>T$ we have $\tilde{\mathcal{F}}(x, u)<0$ and hence (1.2) is oscillatory. 
If instead of the principal solution of (1.2) at $\infty$ we use its nonprincipal solution at $\infty$, then we get the following result.

THEOREM 4.3. Suppose that (4.3) holds, let $\left(\begin{array}{l}x \\ U\end{array}\right)$ be a nonprincipal solution of (1.1) at $\infty$, and let $\left(\begin{array}{l}\bar{x} \\ \tilde{u}\end{array}\right)$ be as in Theorem 4.2. Moreover, we suppose that

$$
\lim _{t \rightarrow \infty} \tilde{x}^{T}(t)\left(\int_{t}^{\infty} \bar{B}(\tau) \Delta \tau\right)^{-1} \tilde{x}(t)=\infty
$$

If

$$
\limsup _{t \rightarrow \infty} \frac{\int^{t}\left\{\tilde{u}^{T} \bar{B} \tilde{u}+\left(\tilde{x}^{\sigma}\right)^{T} \bar{W} \tilde{x}^{\sigma}\right\}(\tau) \Delta \tau}{\tilde{x}^{T}(t)\left(\int_{t}^{\infty} \bar{B}(\tau) \Delta \tau\right)^{-1} \tilde{x}(t)}<-1
$$

then (1.2) is oscillatory.

PROOF. First of all note that since $\left(\begin{array}{l}X \\ U\end{array}\right)$ is the nonprincipal solution of (1.2) at $\infty$, the matrix integral

$$
\int^{\infty} \bar{B}(\tau) \Delta \tau=\int^{\infty}\left\{\left(X^{\sigma}\right)^{-1} B\left(X^{T}\right)^{-1}\right\}(\tau) \Delta \tau
$$

is really convergent [11]. We use again the computations from the proof of Theorem 4.1. For the pair $\left(\begin{array}{l}x \\ u\end{array}\right)$ defined in the proof of that theorem we have

$$
\begin{aligned}
\tilde{\mathcal{F}}(x, u) \leq & \tilde{x}^{T}\left(t_{1}\right)\left(\int_{T}^{t_{1}} \bar{B}(\tau) \Delta \tau\right)^{-1} \tilde{x}\left(t_{1}\right)+\tilde{x}^{T}\left(t_{2}\right)\left(\int_{t_{2}}^{t_{3}} \bar{B}(\tau) \Delta \tau\right)^{-1} \tilde{x}\left(t_{2}\right) \\
& +\int_{t_{1}}^{t_{2}}\left\{\tilde{u}^{T} \bar{B} \tilde{u}+\left(\tilde{x}^{\sigma}\right)^{T} \bar{W} \tilde{x}^{\sigma}\right\}(\tau) \Delta \tau \\
= & \tilde{x}^{T}\left(t_{2}\right)\left(\int_{t_{2}}^{t_{3}} \bar{B}(\tau) \Delta \tau\right)^{-1} \tilde{x}\left(t_{2}\right)\left\{1+\Gamma_{3}+\Gamma_{4}\right\},
\end{aligned}
$$

where

$$
\Gamma_{3}:=\frac{\int_{t_{1}}^{t_{2}}\left\{\tilde{u}^{T} \bar{B} \tilde{u}+\left(\tilde{x}^{\sigma}\right)^{T} \bar{W} \tilde{x}^{\sigma}\right\}(\tau) \Delta \tau}{\tilde{x}^{T}\left(t_{2}\right)\left(\int_{t_{2}}^{t_{3}} \bar{B}(\tau) \Delta \tau\right)^{-1} \tilde{x}\left(t_{2}\right)} \quad \text { and } \quad \Gamma_{4}:=\frac{\tilde{x}^{T}\left(t_{1}\right)\left(\int_{T}^{t_{1}} \bar{B}(\tau) \Delta \tau\right)^{-1} \tilde{x}\left(t_{1}\right)}{\tilde{x}^{T}\left(t_{2}\right)\left(\int_{t_{2}}^{t_{3}} \bar{B}(\tau) \Delta \tau\right)^{-1} \tilde{x}\left(t_{2}\right)}
$$

Let $t_{1}>T$ be fixed and $\varepsilon>0$ be such that the limit superior in (4.10) is less than $-1-3 \varepsilon$. By (4.9) and (4.10), $t_{2}>t_{1}$ can be chosen in such a way that

$$
\frac{\int_{t_{1}}^{t_{2}}\left\{\tilde{u}^{T} \bar{B} \tilde{u}+\left(\tilde{x}^{\sigma}\right)^{T} \bar{W} \tilde{x}^{\sigma}\right\}(\tau) \Delta \tau}{\tilde{x}^{T}\left(t_{2}\right)\left(\int_{t_{2}}^{\infty} \bar{B}(\tau) \Delta \tau\right)^{-1} \tilde{x}\left(t_{2}\right)}<-1-2 \varepsilon
$$


and

$$
\frac{\tilde{x}^{T}\left(t_{1}\right)\left(\int_{T}^{t_{1}} \bar{B}(\tau) \Delta \tau\right)^{-1} \tilde{x}\left(t_{1}\right)}{\tilde{x}^{T}\left(t_{2}\right)\left(\int_{t_{2}}^{\infty} \bar{B}(\tau) \Delta \tau\right)^{-1} \tilde{x}\left(t_{2}\right)}<\varepsilon
$$

Finally, we take $t_{3}>t_{2}$ such that

$$
\Gamma_{3}=\frac{\int_{t_{1}}^{t_{2}}\left\{\tilde{u}^{T} \bar{B} \tilde{u}+\left(\tilde{x}^{\sigma}\right)^{T} \bar{W} \tilde{x}^{\sigma}\right\}(\tau) \Delta \tau}{\tilde{x}^{T}\left(t_{2}\right)\left(\int_{t_{2}}^{t_{3}} \bar{B}(\tau) \Delta \tau\right)^{-1} \tilde{x}\left(t_{2}\right)}<-1-\varepsilon
$$

and also

$$
\Gamma_{4}=\frac{\tilde{x}^{T}\left(t_{1}\right)\left(\int_{T}^{t_{1}} \bar{B}(\tau) \Delta \tau\right)^{-1} \tilde{x}\left(t_{1}\right)}{\tilde{x}^{T}\left(t_{2}\right)\left(\int_{t_{2}}^{t_{3}} \bar{B}(\tau) \Delta \tau\right)^{-1} \tilde{x}\left(t_{2}\right)}<\varepsilon
$$

Consequently, for these $t_{3}>t_{2}>t_{1}>T$ we have $\tilde{\mathcal{F}}(x, u)<0$ and hence (1.2) is oscillatory.

\section{Examples and applications}

In this section we present some corollaries and examples for applications of our general oscillation criteria given in the previous section.

(i) The formulation of Theorems 4.1-4.3 simplifies if the pair $\left(\begin{array}{l}\bar{x} \\ \tilde{u}\end{array}\right)$ appearing in these theorems is of the form $\left(\begin{array}{l}v \\ 0\end{array}\right)$, where $v \in \mathbb{R}^{n}$ is a constant vector. We formulate this simplification for Theorems 4.1-4.2; Theorem 4.3 simplifies accordingly.

COROLLARY 5.1. Suppose that (4.3) holds and let $\left(\begin{array}{l}X \\ U\end{array}\right)$ be as in Theorems 4.1-4.2. If there exists $v \in \mathbb{R}^{n}$ such that

$$
\int^{\infty} v^{T} \bar{W}(\tau) v \Delta \tau=-\infty
$$

or

$$
\int^{\infty} v^{T} \bar{W}(\tau) v \Delta \tau>-\infty \quad \text { and } \limsup _{t \rightarrow \infty} \frac{\int_{t}^{\infty} v^{T} \bar{W}(\tau) v \Delta \tau}{v^{T}\left(\int^{t} \bar{B}(\tau) \Delta \tau\right)^{-1} v}<-1 \text {, }
$$

then (1.2) is oscillatory.

Proof. The statement follows immediately from Theorems 4.1-4.2 taking into account that (4.7) is satisfied for $\tilde{x}(t)=v$ due to the fact that $\left(\begin{array}{l}X \\ v\end{array}\right)$ is the principal solution of (1.1). 
(ii) Here we consider the case $\mathbb{T}=\mathbb{R}$, that is, $\mu \equiv 0$. In this case (1.2) is the linear Hamiltonian system

$$
\left(\begin{array}{l}
x \\
u
\end{array}\right)^{\prime}=\left(\begin{array}{cc}
A & B \\
C+W & -A^{T}
\end{array}\right)\left(\begin{array}{l}
x \\
u
\end{array}\right)
$$

with symmetric matrices $B, C$ and $W$. Oscillatory properties of (5.1) in the case when $A \equiv 0$ (using the variational method presented in Section 4) were investigated in [8]. In that paper only the possibility $\left(\begin{array}{l}\bar{x} \\ \bar{u}\end{array}\right)=\left(\begin{array}{l}v \\ 0\end{array}\right)$ with a constant vector $v \in \mathbb{R}^{n}$ was considered, so the results of Section 4 are new even for linear Hamiltonian differential systems.

(iii) The higher-order Sturm-Liouville differential equation

$$
L(y):=\sum_{\nu=0}^{n}(-1)^{v}\left(r_{\nu}(t) y^{(v)}\right)^{(v)}=0
$$

with $r_{n}(t)>0$ can be written (using a suitable substitution) as the linear Hamiltonian system

$$
x^{\prime}=A(t) x+B(t) u, \quad u^{\prime}=C(t) x-A^{T}(t) u
$$

with

$$
A(t)=\left(a_{i j}\right)_{1 \leq i, j \leq n}, \quad \text { where } \quad a_{i j}= \begin{cases}1 & \text { if } j=i+1,1 \leq i \leq n-1 \\ 0 & \text { otherwise }\end{cases}
$$

and $B(t)=\operatorname{diag}\left\{0,0, \ldots, 0,1 / r_{n}(t)\right\}$ and $C(t)=\operatorname{diag}\left\{r_{0}(t), \ldots, r_{n-1}(t)\right\}$. Oscillatory properties (with applications in the spectral theory of differential operators) of the equation

$$
L(y)+q(t) y=0
$$

viewed as a perturbation of the nonoscillatory equation $L(y)=0$ were investigated in several recent papers, see for example $[9,15]$ and the references given therein. Writing (5.2) as a linear Hamiltonian system (5.1), the perturbation matrix $W$ is of the special form $W=\operatorname{diag}\{0,0, \ldots, 0, q\}$. Using our method, one can investigate oscillatory properties of the equation

$$
L(y)+M(y)=0, \quad \text { where } \quad M(y)=\sum_{\nu=0}^{m}(-1)^{\nu}\left(q_{\nu}(t) y^{(v)}\right)^{(v)}
$$

with $q_{m}(t)>0$ and $m<n$. In this case the perturbation matrix $W$ is

$$
W=\operatorname{diag}\left\{q_{0}, \ldots, q_{m}, 0,0, \ldots, 0\right\} .
$$


If the order of the operator $M$ is higher or equal to that of $L$, that is, $m \geq n$, then this perturbation does not fit into our setting. However, in applications, the perturbation operator is usually of lower order than the original one, and it is also a partial justification why the perturbation matrix $\tilde{\mathcal{S}}$ is of the form as considered here.

As an example of the application of this general idea to fourth-order differential equations we give the following oscillation criterion.

COROLLARY 5.2. Consider the fourth-order differential equation

$$
y^{\prime \prime \prime \prime}-\left(q_{1}(t) y^{\prime}\right)^{\prime}+q_{0}(t) y=0
$$

with $q_{1}(t) \leq 0$ and $q_{0}(t) \leq 9 /\left(16 t^{4}\right)$ eventually. If there exist $c_{1}, c_{2} \in \mathbb{R}$ such that

$$
\int^{\infty}\left\{q_{1}(\tau)\left(h^{\prime}(\tau)\right)^{2}+\left(q_{0}(\tau)-\frac{9}{16 \tau^{4}}\right) h^{2}(\tau)\right\} d \tau=-\infty,
$$

where $h(t)=c_{1} t^{(3-\sqrt{10}) / 2}+c_{2} t^{3 / 2}$, then (5.3) is oscillatory.

PROOF. As the "unperturbed" nonoscillatory equation we take the fourth-order Euler equation

$$
y^{\prime \prime \prime \prime}-\frac{9}{16 t^{4}} y=0
$$

Equation (5.5) has solutions

$$
y_{1}(t)=t^{(3-\sqrt{10}) / 2}, \quad y_{2}(t)=t^{3 / 2}, \quad \tilde{y}_{1}(t)=t^{3 / 2} \log t, \quad \tilde{y}_{2}(t)=t^{(3+\sqrt{10}) / 2} .
$$

By a direct computation one can verify that

$$
X=\left(\begin{array}{ll}
y_{1} & y_{2} \\
y_{1}^{\prime} & y_{2}^{\prime}
\end{array}\right), \quad U=\left(\begin{array}{cc}
-y_{1}^{\prime \prime \prime} & -y_{2}^{\prime \prime \prime} \\
y_{1}^{\prime \prime} & y_{2}^{\prime \prime}
\end{array}\right)
$$

is the principal solution of the linear Hamiltonian system corresponding to (5.5), and (5.3) can be written as a system (5.1) with

$$
A=\left(\begin{array}{ll}
0 & 1 \\
0 & 0
\end{array}\right), \quad B=\left(\begin{array}{ll}
0 & 0 \\
0 & 1
\end{array}\right), \quad C=\left(\begin{array}{cc}
9 /\left(16 t^{4}\right) & 0 \\
0 & 0
\end{array}\right)
$$

and

$$
W(t)=\operatorname{diag}\left\{q_{1}(t), q_{0}(t)-\frac{9}{16 t^{4}}\right\} .
$$

We take $\tilde{x}(t)=c$ and $\tilde{u}(t)=0$ with $c=\left(\begin{array}{l}c_{1} \\ c_{2}\end{array}\right)$ and apply Theorem 4.1. Then

$$
\tilde{x}^{T} W \tilde{x}=c^{T} X^{T} W X c=\left(c_{1} y_{1}^{\prime}+c_{2} y_{2}^{\prime}\right)^{2} q_{1}+\left(c_{1} y_{1}+c_{2} y_{2}\right)^{2}\left(q_{0}-\frac{9}{16 t^{4}}\right),
$$

and (4.5) reduces to (5.4). 
Note that for the sake of simplicity in the previous corollary we used Theorem 4.1 (with the special choices $\tilde{x}=c$ and $\tilde{u}=0$ ). Computing explicitly the expressions

$$
c^{T}\left(\int^{t}\left\{X^{-1} B\left(X^{T}\right)^{-1}\right\}(\tau)\right)^{-1} c \text { and } c^{T}\left(\int_{t}^{\infty}\left\{\tilde{X}^{-1} B\left(\tilde{X}^{T}\right)^{-1}\right\}(\tau)\right)^{-1} c
$$

with

$$
B=\left(\begin{array}{ll}
0 & 0 \\
0 & 1
\end{array}\right) \quad \text { and } \quad \tilde{X}=\left(\begin{array}{cc}
\tilde{y}_{1} & \tilde{y}_{2} \\
\tilde{y}_{1}^{\prime} & \tilde{y}_{2}^{\prime}
\end{array}\right)
$$

(the functions $\tilde{y}_{1}$ and $\tilde{y}_{2}$ are given in the previous proof), one can also formulate oscillation criteria which are special cases of Theorems 4.2-4.3. These reformulations yield new results even for the special equation (5.3).

(iv) Now we deal with the discrete case $\mathbb{T}=\mathbb{Z}$. In this case, (1.2) reduces to the symplectic difference system

$$
z_{k+1}=\left(I+\mathcal{S}_{k}\right) z_{k}
$$

Basic properties of solutions of (5.6) (for example, the Reid roundabout theorem) have been established in $[1,2]$. However, oscillation criteria for general symplectic difference systems (in terms of the coefficient matrices $I+A, B, C$ and $I+D$ of (5.6)) have not been established yet; so the results of Theorems 4.1-4.3 are also new for systems (5.6). We refer here to the papers $[4,7,10,13,15,17]$ and the references contained therein, where oscillatory properties of special cases of (5.6) like discrete Hamiltonian systems or higher-order Sturm-Liouville difference equations are investigated.

\section{Acknowledgement}

Ondřej Došlý was supported by Grant 201/01/0079 of the Grant Agency of the Czech Republic.

\section{References}

[1] M. Bohner, "Linear Hamiltonian difference systems: disconjugacy and Jacobi-type conditions", $J$. Math. Anal. Appl. 199 (1996) 804-826.

[2] M. Bohner and O. Došly, "Disconjugacy and transformations for symplectic systems", Rocky Mountain J. Math. 27 (1997) 707-743.

[3] M. Bohner, O. Došlý and R. Hilscher, "Linear Hamiltonian dynamic systems on time scales: Sturmian property of the principal solution", Nonlinear Anal. 47 (2001) 849-860.

[4] M. Bohner, O. Došlý and W. Kratz, "An oscillation theorem for discrete eigenvalue problems", Rocky Mountain J. Math. 33 (2002) 1233-1260. 
[5] M. Bohner and A. Peterson, Dynamic equations on time scales: an introduction with applications (Birkhäuser, Boston, 2001).

[6] M. Bohner and A. Peterson, Advances in dynamic equations on time scales (Birkhäuser, Boston, 2003).

[7] S. Chen and L. Erbe, "Oscillation results for second order scalar and matrix difference equations", Comput. Math. Appl. 28 (1994) 55-69.

[8] O. Došlý, "On the existence of conjugate points for linear differential systems", Math. Slovaca 40 (1990) 87-99.

[9] O. Došlý, "Oscillation and spectral properties of a class of singular differential operators", Math. Nachr. 188 (1997) 49-68.

[10] O. Došlý, "Oscillation criteria for higher order Sturm-Liouville difference equations", J. Differ. Equations Appl. 4 (1998) 425-450.

[11] O. Doslý, "Principal and nonprincipal solutions of symplectic dynamic systems on time scales", in Proceedings of the 6th Colloquium on the Qualitative Theory of Differential Equations (Szeged, 1999), Electron. J. Qual. Theory Differ. Equ., No. 5, (electronic), (Szeged, 2000) pp. 14.

[12] O. Došlý and R. Hilscher, "Disconjugacy, transformations and quadratic functionals for symplectic dynamic systems on time scales", J. Differ. Equations Appl. 7 (2001) 265-295.

[13] L. Erbe and P. Yan, "On the discrete Riccati equation and its applications to discrete Hamiltonian systems", Rocky Mountain J. Math. 25 (1995) 167-178.

[14] S. Hilger, "Analysis on measure chains - a unified approach to continuous and discrete calculus", Results Math. 18 (1990) 18-56.

[15] R. Hilscher, "Discrete spectral criteria for a certain class of singular differential and difference operators", Comput. Math. Appl. 42 (2001) 465-476.

[16] R. Hilscher, "Reid roundabout theorem for symplectic dynamic systems on time scales", Appl. Math. Optim. 43 (2001) 129-146.

[17] A. Peterson, "Oscillation of second order linear matrix difference equations", J. Differential Equations 89 (1991) 65-88. 